\title{
Effect of mean void fraction correlations on a shell-and-tube evaporator dynamic model
}

\section{performance}

\author{
Joaquín Navarro Esbrí ${ }^{1}$, Víctor Milián ${ }^{2}$, Adrián Mota-Babiloni ${ }^{1,2}$, Francisco Molés ${ }^{1}$, \\ Gumersindo Verdú ${ }^{2}$ \\ ${ }^{1}$ ISTENER Research Group, Department of Mechanical Engineering and Construction, \\ University Jaume I, E12071 Castellón, Spain \\ ${ }^{2}$ Institute for Industrial, Radiophysical and Environmental Safety, Camino de Vera s/n, \\ Polytechnic University of Valencia, E46022 Valencia, Spain
}

\begin{abstract}
In this paper, the influence of different mean void fraction correlations on a shell-and-tube evaporator dynamic model performance has been evaluated. The model proposed is based on the moving boundary approach and includes the expansion valve modelling. Several transient tests, using R134a as working fluid, have been carried out varying refrigerant mass flow, inlet enthalpy and secondary fluid flow. Then, the model performance, using different mean void fractions, is analysed from the system model outputs (evaporating pressure, refrigerant outlet temperature and condensing water outlet temperature). The slip ratio expressions selected are: homogenous, momentum flux model, Zivi's, Chisholm's and Smith's correlations. The results of the comparison between experimental and model predictions depend on the transient characteristics and there is not a single slip ratio correlation that provides the best performance in all the cases analysed.
\end{abstract}

Keywords: evaporator; dynamic model; mean void fraction; slip ratio; refrigeration

\section{Nomenclature}




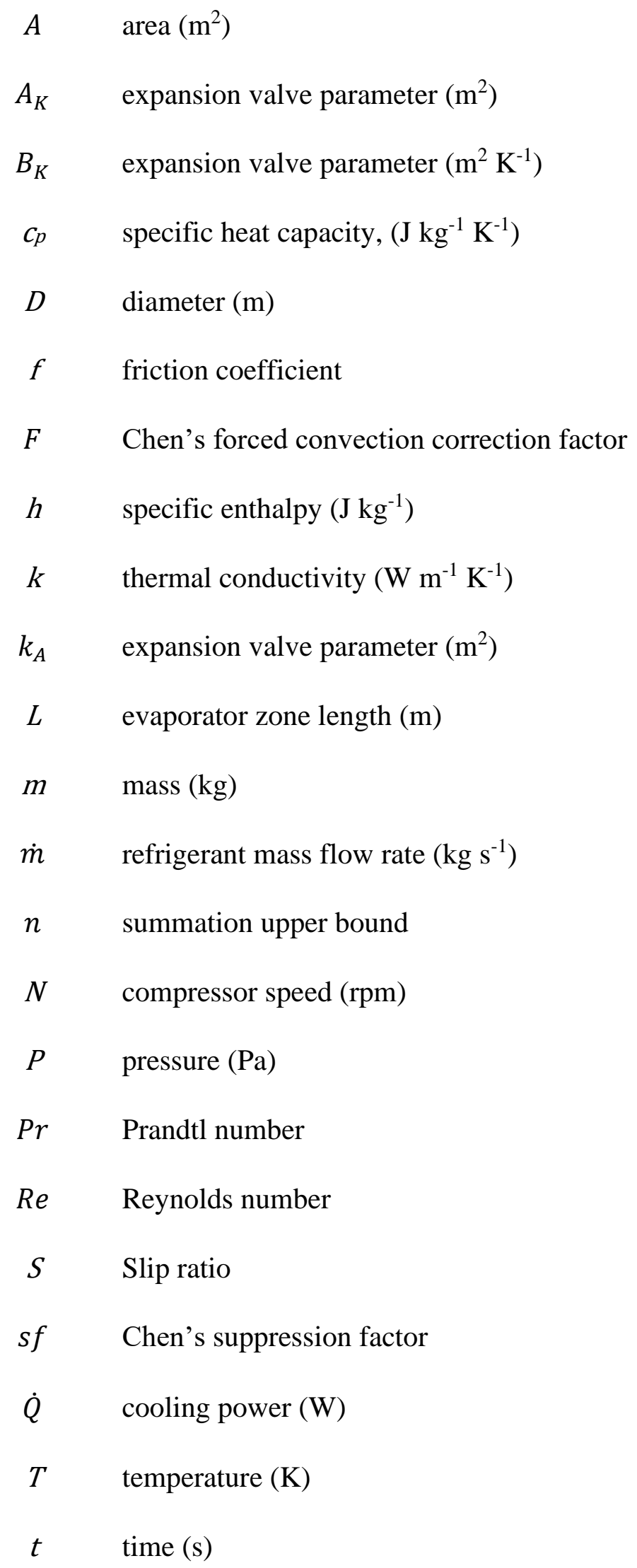




$\begin{array}{cl}u & \text { dynamic viscosity }(\mu \mathrm{Pa} \mathrm{s}) \\ \dot{V} & \text { volumetric flow rate }\left(\mathrm{m}^{3} \mathrm{~s}^{-1}\right) \\ X & \text { vapour quality } \\ X_{t t} & \text { Martinelli parameter }\end{array}$

Greek symbols

$\begin{array}{cl}\alpha & \text { heat transfer coefficient }\left(\mathrm{W} \mathrm{m}^{-2} \mathrm{~K}^{-1}\right) \\ \gamma & \text { mean void fraction } \\ \Delta T & \text { degree of superheating }(\mathrm{K}) \\ \Delta T_{\text {static }} & \text { static degree of superheating }(\mathrm{K}) \\ \mu & \text { density ratio } \\ \rho & \text { density }\left(\mathrm{kg} / \mathrm{m}^{3}\right) \\ \sigma & \text { vapour-liquid surface tension }\left(\mathrm{N} \mathrm{m}^{-1}\right) \\ v & \text { specific volume }\left(\mathrm{m}^{3} \mathrm{~kg}^{-1}\right)\end{array}$

Subscripts

$\begin{array}{cl}\text { actual } & \text { experimental value } \\ b f & \text { two-phase } \\ c & \text { condensing } \\ \text { cat } & \text { catalogue } \\ \text { Ch } & \text { Chisholm's correlation } \\ \text { conv } & \text { convective }\end{array}$




\begin{tabular}{|c|c|}
\hline CS & cross section \\
\hline$e$ & evaporator \\
\hline$e x$ & external \\
\hline$g$ & glycol-water mixture \\
\hline$h$ & homogenous model \\
\hline$i$ & inlet \\
\hline in & internal \\
\hline$k$ & $k$-value of a data set \\
\hline$L$ & saturated liquid \\
\hline$L V$ & liquid to vapour \\
\hline$M$ & metal surface \\
\hline $\max$ & maximum \\
\hline $\min$ & minimum \\
\hline$M F$ & momentum flux model \\
\hline$n b$ & nucleate boiling \\
\hline$r$ & refrigerant \\
\hline$s$ & shell \\
\hline$S m$ & Smith's correlation \\
\hline$t$ & tube \\
\hline$T e$ & total evaporator length \\
\hline$o$ & outlet \\
\hline$V$ & saturated vapour \\
\hline$V S$ & vapour to superheating \\
\hline$Z$ & Zibi's correlation \\
\hline
\end{tabular}


1e evaporation zone

$2 e \quad$ superheating zone

Acronyms

$\begin{array}{ll}\text { FV } & \text { finite-volume distributed-parameter model } \\ \text { MB } & \text { moving-boundary model } \\ \text { MVF } & \text { mean void fraction } \\ \text { RMS } & \text { Root mean square value }\end{array}$

\section{Introduction}

Refrigeration facilities based on vapour compression cycles are responsible for about $30 \%$ of the total energy consumption (Bouzelin et al., 2005) for utility companies. Therefore, it is desirable to use appropriate models to improve both their performance and management. Most of the models used, either steady state or dynamic, are based on physical laws and they usually use fluid properties and components characteristics as input data.

When the aim of the model is simply to simulate specific conditions or to design systems and components, the steady state modelling is enough. In the available literature there are a lot of works that refer to steady state models of vapour compression systems (Gordon and NG, 2000), applied to different type of installations, as reciprocating (Bourdouxhe et al., 1994) or centrifugal (Braun et al., 1996) chillers. Browne and Bansal (1998) also reported different models of vapour-compression liquid chillers developed in the past decades and Li et al. (2014) reviewed the research advancement in dynamic modeling of HVAC equipment. 
As vapour compression systems work most of the time under transient conditions (Roetzel and Xuan, 1999), steady state models cannot accurately describe the system response due to variations in their operating variables. Therefore, sometimes it is necessary to characterize the system transient behaviour by means of dynamic models. In this way, the characteristics of the systems can be better analysed, the new components can be properly designed and, finally, that could lead to improve its operation and efficiency. Bendapudi and Braun (2002) summarized various methodologies adopted in transient modelling and their applicability to chillers.

It is well-known that heat exchangers, and the types of changes related to their dynamics (Rasmussen and Shenoy, 2012), are the most complex parts of the vapour compression models. To describe the dynamic behaviour of heat exchangers three main approaches are commonly used (Rasmussen, 2012): finite-volume distributed-parameter (FV), movingboundary lumped-parameter (MB) models and a hybrid technique of both. The general methodology applied in these approaches consists of applying the conservation equations into the heat exchanger control volumes.

On the one hand, when using the MB model, each control volume corresponds to those of the different fluid phase regions. In refrigeration and air conditioning evaporators, two zones are considered: evaporation zone and vapour superheating zone. The limits of those regions are the moving boundaries that determine their lengths, which in turn are dynamic variables. On the other hand, when using the FV model, the heat exchanger is divided in control volumes of a constant size. 
Although FV models can be more accurate (MacArthur and Grald, 1989, Cullimore and Hendriks, 2001, Eborn et al., 2005 and Limperich et al., 2005) than MB models, they can require up to 15 control volumes to obtain good results (Bendapudi et al., 2008). This occurs because there are much more conservation equations than in the MB approach, resulting in a lower execution speed. MB models can be developed about three times faster than FV models and that is very important for control and diagnostic purposes (Bendapudi et al., 2008, Bendapudi, 2004).

The MB model was first pioneered by Wedekind et al. (1978). This approach uses the concept of a mean void fraction (MVF), calculated from the local void fraction and defined as the cross-sectional area occupied by the vapour in relation to the total area of the flow channel. This parameter can be calculated through different correlations and geometric definitions: local, chordal, volumetric and cross-sectional (commonly used for two-phase flow) (Collier and Thome, 1994 and Thome, 2004).

Extensive lists of void fraction models and correlations for internal flow are given by Rice (1987), Woldesemayat and Ghajar (2007), and Dalkilic et al. (2008). Among them, one of the most common are the slip ratio correlations, where the void fraction depends on the vapour quality and some fluid properties (Wallis, 1969). Dalkilic et al. (2008) realized a comparison in a vertical smooth tube (in steady state flow) and concluded that most of the slip ratio correlations have results that are compatible with each other for the same operating conditions. Milian et al. (2013) developed a dynamic model of a shell-and-tube condenser and studied their performance using different mean void fraction correlations. In the dynamic model (using R407C) of Haberschill et al., (2003) was simulated the control of cooling capacity by opening the expansion valve and by varying the compressor speed. 
The aim of this paper is to develop of a MB dynamic model of a Direct Expansion evaporator (including the expansion valve model) evaluating the influence of different slip ratio correlations on the model performance. The evaluation is quantified comparing the model predictions and the experimental data measurements for different transient situations in a vapour compression system using R134a as refrigerant.

The rest of the paper is organized as follows. In Section 2, the proposed model is described. In Section 3, the correlations used to obtain the different mean void fractions are presented. In Section 4, the experimental test bench and tests used to validate the model are briefly explained. In section 5 the results are showed and, finally, in section 6 the main conclusions of this work are summarized.

\section{Model description}

An overview of the structure of the proposed model is shown in Fig. 1.

Fig. 1. Model scheme.

The model takes five parameters as input variables: refrigerant mass flow rate, $\dot{m}_{r}$, evaporator inlet refrigerant enthalpy, $h_{i e}$, propylene glycol-water mixture mass flow rate and temperature, $\dot{m}_{g}$ and $T_{g, i e}$, respectively, and expansion valve static superheating degree, $\Delta T$. 
The model outputs are: length of evaporating zone, $L_{1 e}$, evaporating pressure, $P_{e}$, evaporator outlet refrigerant enthalpy, $h_{o e}$, and tube wall temperatures in evaporation zone $(1 e)$ and superheating zone $(2 e), T_{t, 1 e}$ and $T_{t, 2 e}$, respectively. From these outputs it can be easily derived the other measurable outputs, refrigerant outlet temperature, $T_{r, o e}$, and glycol-water outlet temperature, $T_{g, o e}$.

The expansion valve and evaporator model equations, the mean void fractions expressions used and the applied heat transfer coefficients are presented below.

\subsection{Expansion valve}

According to previous studies (Rasmussen, 2005), the expansion valve mechanical dynamics are significantly faster than the expansion valve thermal dynamics, being the latter similar to the vapour compression system dynamics. Due to this difference, the valve is modelled with static relationships.

Under normal operation the mass flow rate through the component is a fraction of the maximum value given by the manufacturer's catalogue and is given by Eq. (1) (Belman et al., 2010).

$$
\dot{m}_{r}=\dot{m}_{r, \text { cat }} \frac{\Delta T_{\text {static }}-\Delta T}{\Delta T_{\text {static,max }}-\Delta T}
$$

$\dot{m}_{r, c a t}$ is given in the Eq. (2). 


$$
\dot{m}_{r, c a t}=k_{A} \sqrt{\rho_{L}\left(P_{c}-P_{e}\right)}
$$

$k_{A}$ is a parameter characterized by a general correlation (Saiz Jabardo et al., 2002) presented in Eq. (3).

$$
k_{A}=A_{k}+B_{k} T_{e}
$$

$A_{k}$ and $B_{k}$ depend on the valve chosen. For the current valve are equal to $2.433 \cdot 10^{-6} \mathrm{~m}^{2}$ and 4.857.10 $10^{-8} \mathrm{~m}^{2} \mathrm{~K}^{-1}$ (Belman et al., 2010).

The initial value of static superheating degree is obtained from manufacturer's data. Besides, an experimental correlation for the maximum superheating degree in terms of the static superheating degree is given by Eq. (4) (Belman et al., 2010).

$$
\Delta T_{\text {static, } \max }=-0.75+1.75 \Delta T
$$

o

\subsection{Evaporator}

For modelling purposes, the refrigerant flow in the shell-and-tube evaporator (Fig. 2a) can be approached to an equivalent axial tube heat exchanger (Fig. 2b) (Grald and MacArthur, 1992). Thus, it can be considered that the glycol water mixture flows through the outer tube and the inner tube carries the refrigerant. 
Fig. 2. Shell-and-tube evaporator inner structure and fluid path (a), and equivalent axial tube with two evaporator zones (b).

As can be seen in Fig. 2, the evaporator is represented with two zones: evaporation zone and superheating zone, whose lengths are the model outputs $L_{1 e}$ (being also the moving boundary) and $L_{2 e}$, respectively.

In what follows, the governing partial differential equations are described, as well as the way to obtain the governing ordinary differential equations of the lumped parameter model.

The main simplifying assumptions of the model are as follow:

- The mass flow rate of the refrigerant is assumed to be the same throughout the two components of the subsystem.

- The fluid flow in the evaporator is one-dimensional.

- Pressure drops are negligible.

- The expansion process through the thermostatic expansion valve is isenthalpic.

- There is no axial heat transfer conduction in the fluid flow.

- There is no axial heat transfer conduction in the tube wall, and there is no wall temperature variation along its cross section.

- Heat conduction through the shell can be neglected.

The evaporator zones can be modelled from the Navier-Stokes generalized equations (Willatzen et al., 1998) and from the energy conservation equation in the evaporator's wall. 
Due to the simplifying assumptions, the momentum equation can be eliminated (Eq. (5), (6) and (7)).

$$
\begin{array}{cc}
\frac{\partial \rho A_{c s}}{\partial t}+\frac{\partial \dot{m}_{r}}{\partial z}=0 & \text { Refrigerant mass balance } \\
\frac{\partial\left(\rho A_{c s} h-A_{c s} P_{e}\right)}{\partial t}+\frac{\partial\left(\dot{m}_{r} h\right)}{\partial z} & \\
=\alpha_{i n} \pi D_{i n}\left(T_{t}-T_{r}\right) & \text { Refrigerant energy balance } \\
m_{t e} c_{p, t} \frac{\partial T_{t}}{\partial t}=\alpha_{i n} \pi D_{i n} L\left(T_{r}-T_{t}\right) & \\
+\alpha_{e x} \pi D_{e x} L\left(T_{g}-T_{t}\right) & \text { Tube wall energy balance }
\end{array}
$$

The spatial dependence of the previous partial differential equations is taken away by integrating the equations over the length of each region (Zhang et al., 2006). By applying Leibnitz's rule on the first terms and integrating the second terms, a set of six ordinary differential equations is obtained. The integration of Eq. (5) and (6) in the two-phase flow region requires to use the concept of mean void fraction, $\gamma$, to characterize the density and enthalpy, Eq. (8) and (9).

$$
\begin{gathered}
\rho=\rho_{L}(1-\gamma)+\rho_{V} \gamma \\
\rho h=\rho_{L} h_{L}(1-\gamma)+\rho_{V} h_{V} \gamma
\end{gathered}
$$

The six aforementioned ordinary differential equations are reduced to five after removing the refrigerant flow rate at the intermediate point, $\dot{m}_{i n t}$, where vapour quality is 1 . The resulting 
equations are shown in a compact form in Eq. (10) and the equations terms $z_{i j}$ and $y_{i j}$ are given in Table 1 and Table 2.

$$
\left[\begin{array}{ccccc}
z_{11} & z_{12} & 0 & 0 & 0 \\
z_{21} & z_{22} & z_{23} & 0 & 0 \\
z_{31} & z_{32} & z_{33} & 0 & 0 \\
0 & 0 & 0 & z_{44} & 0 \\
z_{51} & 0 & 0 & 0 & z_{55}
\end{array}\right]\left[\begin{array}{c}
\dot{L}_{1 e} \\
\dot{P}_{e} \\
\dot{h}_{o} \\
\dot{T}_{t, 1 e} \\
\dot{T}_{t, 2 e}
\end{array}\right]=\left[\begin{array}{c}
y_{11}+\rho_{L}\left(h_{L}-h_{V}\right) A_{c s, e} \dot{\gamma} L_{1 e} \\
y_{12}-\left(\rho_{V}-\rho_{L}\right) h_{V} A_{c s, e} \dot{\gamma} L_{1 e} \\
y_{13}-\left(\rho_{V}-\rho_{L}\right) A_{c s, e} \dot{\gamma} L_{1 e} \\
y_{14} \\
y_{15}
\end{array}\right]
$$

Table 1 . Terms $z_{i j}$ in system model.

Table 2. Terms $y_{i j}$ in system model.

In this model, the enthalpy in the superheating zone, $h_{2 e}$, has been taken as the mean value between input and output enthalpy values of the zone. Then, all the other refrigerant properties are calculated from the pressure and mean enthalpy obtained in each iteration step. Refrigerant properties as well as their time derivatives have been obtained from fitting equations derived from the values provided by REFPROP library (Lemmon et al., 2002), what reduces the execution time in about three times.

\subsection{Heat transfer coefficients}


To obtain the heat transfer coefficients, $\alpha$, there are many expressions available in the open literature (Bendapudi and Braun, 2002) for each different heat exchanger geometric configurations. In this case, as the model is intended to be used in both steady state and dynamic situations, it has been chosen those expressions which have shown a good performance in previous works such as entire vapour compression models (Navarro-Esbrí et al., 2010) or shell-and-tube evaporator models (Navarro-Esbrí et al., 2014). The correlations selected and the equations used are expressed in Table 3.

Table 3. Equations used to obtain the heat transfer coefficients.

\subsection{Mean void fraction correlations}

The mean void fraction and its time derivative are included in the $z_{i j}$ coefficients (coefficient matrix) of the previous model, Eq. (10). Although there are different correlations for predicting void fraction in refrigeration applications (Wilson et al., 1998), in this work it is chosen the local slip ratio (which can be correlated with different void fraction expressions). The equation to calculate this parameter is shown in Eq. (11).

$$
\gamma_{\text {local }}=\frac{x}{x+(1-x) \mu S}
$$

where $\mu=\left(\rho_{V} / \rho_{L}\right)$ and $S$ is the slip ratio (or velocity ratio), which is defined as the ratio between the vapour and liquid speeds in the two-phase region (Thome, 2004). 
If $S=1$ is selected, the homogeneous model is obtained $\left(S_{h}\right)$. However, some authors note that it can be an inadequate representation and propose a slip flow two-phase model with $S>1$ because it provides improved experimental results (Jakobsen et al., 1999).

The analytical void fraction models assume that some quantities, such as momentum or kinetic energy of the two phases, tend towards a minimum value. So, when the momentum flux is assumed to have a minimum value $\left(S_{M F}\right)$ the velocity ratio $S$ is given by Eq. (12).

$$
S_{M F}=\left(\frac{\rho_{V}}{\rho_{L}}\right)^{-1 / 2}
$$

Another frequently used expression to calculate $S$ is proposed by Zivi $\left(S_{Z}\right)$ (Rice, 1987), Eq. (13). This model was proposed for annular flow, with the assumption that no liquid is entrained in the central vapour core and that the total kinetic energy of the two phases will tend to be a minimum.

$$
S_{Z}=\left(\frac{\rho_{V}}{\rho_{L}}\right)^{-1 / 3}
$$

Aside from the previous analytical models, two empirical correlations obtained by Smith and Chisholm are also used in this model (Collier and Thome, 1994). These correlations depend on both evaporating pressure and vapour quality.

The Smith separated flow model provides the following slip ratio expression $\left(S_{S m}\right)$, Eq. (14). 


$$
S_{S m}=e+(1-e)\left[\frac{\frac{\rho_{L}}{\rho_{V}}+e\left(\frac{1-x}{x}\right)}{1+e\left(\frac{1-x}{x}\right)}\right]^{1 / 2}
$$

where $e$ represents the fraction of liquid entrained in the gas as droplets. Eq. (14) is based on the assumption that the momentum fluxes in both phases are equal (Rice, 1987).

The Chisholm correlation $\left(S_{C h}\right)$ is derived from simple annular flow theory and equates the frictional pressure drops in the liquid and the gas phase, Eq. (15).

$$
S_{C h}=\left[1-x\left(1-\frac{\rho_{L}}{\rho_{V}}\right)\right]^{1 / 2}
$$

So, in this paper, five slip ratio correlations are used: $S_{h}, S_{M F}, S_{Z}, S_{S m}, S_{C h}$. These expressions can be used in Eq. (11) to obtain the mean void fraction.

In this way, after using $S_{M F}$ or $S_{Z}$ in Eq. (11) and integrating along the two-phase zone, it can be obtained the mean void fraction, $\gamma(x, S)$, Eq. (16). This expression depends on both the vapour quality and on the slip ratio correlation used (Fig. 3 represents the mean void fraction values obtained when $S=S_{Z}$, varying $\mu$ and the vapour inlet quality).

$$
\gamma=\frac{1}{(1-\mu S)}\left\{1+\frac{\mu S}{\left(x_{2 e}-x_{1 e}\right)(1-\mu S)} \ln \left[\frac{\mu S+x_{1 e}(1-\mu S)}{\mu S+x_{2 e}(1-\mu S)}\right]\right\}
$$


Fig. 3. Quality based mean void fraction, $S=S_{Z}$.

However, when substituting $S_{S m}$ or $S_{C h}$ in Eq. (11), the resulting expression cannot be solved analytically. Instead, a numerical integration can be performed as proposed in Eq. (17). $n$ is the total amount of nodes, in this case it is equal to 10 in order to have enough accuracy (Zhang and Zhang, 2006).

$$
\gamma=\frac{1}{n} \sum_{k=1}^{n}\left(\gamma_{\text {local }}\right)_{k}
$$

The time derivative $\dot{\gamma}$ (included in the right hand terms of the Eq. (10)) can be neglected in this case. This is because the mean void fractions calculated take almost constant values (this can be seen for $S=S_{Z}$ in Fig. 3, particularly for $\mu<0.01$ ).

The slip ratios $S_{M F}$ and $S_{Z}$ only depend on the evaporating pressure, and are calculated straightforward at each program step. But as $S_{S m}$ and $S_{C h}$ also depend on the vapour quality at each point of the evaporating zone, their mean values must be calculated at each time step along this zone; this is performed approximately by means of numeric integration.

\section{Experimental plant and tests}

The experimental plant is composed of a vapour compression system, using R134a as working fluid, and two secondary fluid circuits (Fig. 4). The main circuit consists of an open type variable speed compressor, a shell-and-tube condenser, with refrigerant flowing along the shell and the water inside the tubes; a thermostatic expansion valve, and a DX shell-and- 
tube evaporator thermally isolated, where the refrigerant flows inside the tubes (Table 4) and the secondary fluid along the shell.

Fig. 4. Simplified vapour compression plant scheme.

Table 4. Tube characteristics.

The evaporator secondary fluid circuit is a load simulation system (it makes possible to control the evaporator conditions). It consists of a tank with electrical resistances allowing the control of the evaporator's thermal load by means of a variable speed pump and a PID temperature controller. The secondary fluid is a water-propylene glycol (70/30 by mass percentage).

The experimental setup is fully instrumented to measure key variables (their location can be seen at Fig 4.) Table 5 shows a summary of the measured parameters, the sensors used and the uncertainty associated. The signals generated are gathered with a data acquisition system and monitored and controlled through a personal computer.

Table 5. Measured parameters and equipment uncertainty. 
In order to carry out a proper model validation, the transient tests were realised over a wide range of operating conditions (Table 6). In each transient test, only one input changes: mass flow rate, evaporator inlet enthalpy or glycol-water mixture flow rate. First, these parameters are lowered (or increased) and subsequently are increased (or lowered) in about the same amount. The static superheating degree is maintained constant at $5 \mathrm{~K}$, and the rest of the inputs remain almost stable (their mean values and standard deviation are also shown).

Table 6. Parameters changes that originates the transients.

\section{Results and discussion}

In this section the comparison between experimental and model predictions using different slip ratio correlations (mentioned in brackets for each parameter considered) is presented. Thus, the predicted model outputs (evaporating pressure, refrigerant outlet temperature and glycol-water outlet temperature), using different slip ratios correlations, are compared with the experimental measurements (Fig. 5 to Fig. 10). It is also shown how the MVF corresponding to those slip ratios behave over the course of the tests.

As said before, the model inputs are incremented and decremented in the same amount. But since the trends are similar, it is only shown the model outputs for one performed change (either decrease or increase). 
The root mean square (RMS) deviation is used to quantify the difference between each value predicted by the model $X_{S}$ and the experimental value measured $X_{\text {actual }}$, Eq. (18).

$$
R M S(X)=\sqrt{\frac{\sum_{k=1}^{n}\left(X_{a c t u a l, k}-X_{S, k}\right)^{2}}{n}}
$$

$X$ represents the outputs $P_{e}, T_{r, o e}, T_{g, o e}$ and the subindex $S$ represents the slip ratio expression used, described above $(S=h, M F, Z, S m, C h) . k$ is the $k$-value of the data set and model results and $n$ represents the total amount of measurements in each test. The RMS values obtained with each void fraction correlation are given in Table 7.

Table 7. RMS values.

In the following paragraphs, the system responses due to changes in input variables (refrigerant mass flow rate, evaporator inlet quality or glycol-water mixture flow rate) are analysed.

\subsection{Changes in refrigerant mass flow rate}

The changes in refrigerant mass flow rates are contemplated in tests from 1 to 6 , with small changes (test 1 and test 2), medium changes (test 3 and test 4) and high changes (test 5 and test 6). 
As shown in Table 6, the refrigerant mass flow rate in test 1 is changed from 0.0563 to 0.0548 $\mathrm{kg} \mathrm{s}^{-1}$, and in test 2 it has been changed from 0.0555 to $0.0569 \mathrm{~kg} \mathrm{~s}^{-1}$. Both variations can be considered as small changes. The transient responses of the system model outputs for test 1 are shown in Fig. 5.

Fig. 5. Test 1. Model outputs due to small decrease in refrigerant mass flux, (a) evaporating pressure, (b) refrigerant outlet temperature, (c) secondary fluid outlet temperature.

As can be seen in Fig. 5, the model correctly predicts the dynamics of the three outputs. The model results are very similar for $P_{e}$ and $T_{r, o e}$ (except with the homogeneous model) and converge quite well to the experimental values (being particularly good for $T_{g, o e}$ ), although a slight slower response in pressure predictions is detected. As the trend and dynamics of all outputs in test 2 are similar to those in test 1 , the test 2 graphics are not shown in this paper.

From the RMS values given in Table 7, it can be deduced that the slip ratio that produces best model performance, in terms of RMS values, in both tests is $S_{M F}$ for $P_{e}$ and $T_{r, o e}$ (except in Test 2, where $S_{Z}$ is a slight better), being the model predictions obtained using $S_{C h}, S_{S m}$ and $S_{Z}$ very close. The slip ratio that produces the highest values of RMS in the evaporating pressure and refrigerant outlet temperature is the homogeneous model.

From Table 7 it can also be seen that for $T_{g, o e}$, the RMS differences are almost negligible. 
The next group of transient responses are obtained with medium changes in refrigerant mass flow. Test 3 when it drops from 0.0556 to $0.0503 \mathrm{~kg} \mathrm{~s}^{-1}$ and test 4 when it increases in about the same quantity from 0.0483 to $0.0543 \mathrm{~kg} \mathrm{~s}^{-1}$. In these two tests the changes are higher than previously. The model outputs transients for test 3 are shown in Fig. 6.

Fig. 6. Test 3. Model outputs due to medium decrease in refrigerant mass flux, (a) evaporating pressure, (b) refrigerant outlet temperature, (c) secondary fluid outlet temperature.

From Fig. 6 it can be seen that the model predicts correctly enough the dynamics of the three outputs, being the homogeneous correlation the one that gives higher errors in model predictions. The output trends are similar in both tests 3 and 4. All RMS values in tests 3 and 4 are greater than in tests 1 and 2.

According to the RMS values, the best performing slip ratios, in terms of RMS values, for $P_{e}$ and $T_{r, o e}$ in both tests are $S_{C h}$ and $S_{S m}$ (and their results are very similar). Equal to tests 1 and 2 , the worst values are given by $S_{h}$.

Fig. 6c shows that $T_{g, o e}$ follows and approaches very well to the experimental values for all correlations. The maximum relative error in both tests is $0.13 \%$ for $T_{g, o e}\left(S_{C h}\right)$.

In test 5 and 6 the highest changes in mass flow rate are performed: in test 5 it drops from $0.0569 \mathrm{~kg} \mathrm{~s}^{-1}$ to $0.0497 \mathrm{~kg} \mathrm{~s}^{-1}$ and in test 6 it increases in a similar quantity, from 0.0497 $\mathrm{kg} \mathrm{s}^{-1}$ to $0.0603 \mathrm{~kg} \mathrm{~s}^{-1}$. Fig. 7 shows the behavior of the three model outputs in test 5 . The 
transients of these model outputs also follow the experimental trends, particularly $T_{g, o e}$. The other two outputs, i.e., $P_{e}$ and $T_{r, o e}$, depart from the experimental values even more than in previous tests. RMS results for each slip ratio correlation are now even higher than in previous tests.

Fig. 7. Test 5. Model outputs due to high decrease in refrigerant mass flux, (a) evaporating pressure, (b) refrigerant outlet temperature, (c) secondary fluid outlet temperature.

For these two tests, the RMS results for $P_{e}$ and $T_{r, o e}$ are very similar to the previous tests. The empirical correlations of Smith and Chisholm are the best performing and, again, the worst performing correlation is $S_{h}$.

As in previous tests, the model using any of the proposed slip ratio correlations follows and approaches very well to the experimental values for all correlations.

\subsection{Changes in evaporator inlet enthalpy}

The changes in evaporator inlet enthalpy are represented by tests from 7 to 10 . Test 7 and test 8 show small changes and test 9 and test 10 present higher changes.

Analysing the small changes, in test 7 (Fig. 8), evaporator inlet enthalpy increases from about 251 to $258 \mathrm{~kJ} \mathrm{~kg}^{-1}$, whereas in test 8, the inlet enthalpy drops from 256 to $251 \mathrm{~kJ} \mathrm{~kg}^{-1}$. 
Fig. 8. Test 7. Model outputs due to small decrease in evaporator inlet enthalpy, (a) evaporating pressure, (b) refrigerant outlet temperature, (c) secondary fluid outlet temperature.

Using all the slip ration correlations (except the homogenous one), the model outputs follow quite well the experimental values. As in all cases seen up till now, the homogeneous model predicts a lower pressure and a higher temperature than the experimental values, so both departs significantly.

The model predictions using $S_{C h}, S_{S m}$ and $S_{Z}$ are very next to the actual values for $P_{e}$ and $T_{r, o e}$. Even $S_{Z}$ is giving good model predictions. About $T_{g, o e}$ model predictions, again all the MVF correlations provide good results as it can be seen also in Fig 8.c.

Regarding to higher changes in evaporator inlet enthalpy, represented by test 9 and test 10 , Fig. 9 shows the results of test 9. It can be seen as the results with the homogeneous correlation depart significantly from the other (as in previous tests). Comparing these results with those obtained in the previous test which evaporator inlet enthalpy is varied (7 and 8), it can be seen as the model outputs are closer to experimental values when inlet enthalpy variations are small. Besides, predictions are better when inlet enthalpy decreases. This can be stated analysing the RMS values. 
Fig. 9. Test 9. Model outputs due to high decrease in evaporator inlet enthalpy, (a) evaporating pressure, (b) refrigerant outlet temperature, (c) secondary fluid outlet temperature.

According to the RMS values obtained for $P_{e}$ in both tests 9 and 10, the best performing slip ratio is given by the homogeneous correlation. The maximum relative errors are observed in $P_{e}\left(\mathrm{~S}_{\mathrm{Ch}}\right)$ and are: $3.30 \%$ and $2.37 \%$ in tests 9 and 10 , respectively. RMS values in test 9 are higher than in test 7 and RMS values in test 10 are higher than in test 8 . Thus, as it happens in the case of refrigerant mass flux changes, enthalpy variations also affect model deviations from the experimental values.

For $T_{r, o e}$, as well as in tests 7 and 8 , the homogeneous correlation is the worst performing with relative errors of $0.46 \%$ and $0.22 \%$ for $T_{r, o e}\left(S_{C h}\right)$ in test 9 and 10 . For $T_{g, o e}$, maximum relative errors are less than $0.1 \%$ for $T_{g, o e}\left(S_{Z}\right)$ in both tests 9 and 10 .

\subsection{Changes in glycol-water mixture flow}

The next set of transient tests is the result of changes in propylene glycol water mixture flow (tests 11 and 12). In Fig. 10 the model outputs of test 11 are represented, which corresponds to a sharp glycol-water flow increase of $2.310^{-4} \mathrm{~m}^{3} \mathrm{~s}^{-1}$, (in test 12 a similar drop in glycolwater flow takes place). Once more the model outputs trends follow quite well the experimental values, and results with the homogeneous model depart significantly from the others, the latter being quite similar to each other. 
Fig. 10. Test 11. Model outputs due to sharp decrease in evaporator secondary fluid flow, (a) evaporating pressure, (b) refrigerant outlet temperature, (c) secondary fluid outlet temperature.

The model predictions for $P_{e}$ and $T_{r, o e}$ in tests 11 and 12 are worst that those obtained in all the previous tests, being the RMS values very similar for all the slip ratio correlations. The homogeneous model continues producing a model overestimation in refrigerant outlet temperature and an underestimation in evaporating pressure in comparison to the other MVF correlations.

Again, $T_{g, o e}$ is predicted accurately using any of the correlations proposed in this work.

\section{Conclusions}

This paper presents a dynamic lumped parameter model for an evaporator with thermostatic expansion valve, using R134a as working fluid. The heat exchanger is modelled with the moving-boundary formulation whereas the expansion valve is described by a steady state model. In this model, several slip ratio correlations, used to calculate the mean void fraction parameter, have been used, and their influence on the model results has been analysed, comparing model predictions with experimental data measured from transient tests.

In general, the physical model used in this work predicts the evaporating pressure with a maximum relative error of $\pm 8 \%$, and the refrigerant and evaporator secondary fluid output temperatures with maximum relative errors always below 2\%. As these errors usually 
correspond to the worst performing slip ratios of each case, smaller errors are obtained with other slip ratio correlations.

The influence of the slip ratio correlations on the model accuracy depends on the output considered, the transient situation chosen. For refrigerant mass flow rate variations, in general, the most accurate model predictions are obtained when Chisholm's or Smith's correlations are used. For evaporator inlet enthalpy changes Chisholm's or Smith's produce good model predictions, and Zivi's correlation for high changes. For secondary fluid flow variations, the results provided by Chisholm's and Smith's correlations and by the momentum flux model are very similar.

\section{References}

Belman, J.M., Navarro-Esbrí, J., Ginestar, D. and V. Milián, 2010. Steady-state model of a variable speed vapour compression system using R134a as a working fluid. International Journal of Energy Research 34(11): 933-945.

Bendapudi, S. 2004. Development and evaluation of modeling approaches for transients in centrifugal chillers. $\mathrm{PhD}$ thesis, Purdue University.

Bendapudi, S. and J.E. Braun, 2002. A Review of Literature on Dynamic Models of vapour Compression Equipment, ASHRAE Report; No. 4036-5.

Bendapudi, S., Braun, J.E. and E.A. Groll, 2008. A comparison of moving-boundary and finite-volume formulations for transients in centrifugal chillers. International Journal of Refrigeration 31: 1437-1452.

Bourdouxhe, J.P., Grodent, M., Lebrun, J.J. and C.A. Saavedra, 1994. A toolkit for primary HVAC system energy calculation - part 2: reciprocating chiller models, ASHRAE Transactions 100: 774-786.

Bouzelin, L.O.S., Amico, S.C., Vargas, J.V.C. and J.A.R. Parise, 2005. Experimental development of an intelligent refrigeration system. International Journal of Refrigeration 28: 165-175.

Braun, J.E., Mitchell, J.W., Klein, S.A. and W.A. Beckman, 1996. Models for variable speed centrifugal chillers. System simulation in buildings, in: Proceedings of the International Conference in Liege, Belgium pp. 83-111. 
Browne, M.W. and P.K. Bansal, 1998. Challenges in modeling vapour compression liquid chillers. ASRHAE Transactions 104 (Part 1A): 474-486.

Chen, J.C. 1966. A correlation for boiling heat transfer of saturated fluids in convective flow. Industrial and Engineering Chemistry Process Design and Development 5: 322-329.

Collier, J.G. and J.R. Thome, 1994. Convective Boiling and Condensation. Clarendon Press. Oxford.

Cullimore, B.A. and T.J. Hendricks, 2001. Design and transient simulation of vehicle air conditioning systems. SAE Paper 2001-01-1692.

Dalkilic, A.S., Laohalertdecha, S. and S. Wongwises, 2008. Effect of void fraction models on the two-phase friction factor of R134a during condensation in vertical downward flow in a smooth tube. International Communications in Heat and Mass Transfer 35: 921-927.

Dittus, F.W. and L.M.K. Boelter, 1930. Publications on Engineering, vol. 2. University of California: Berkeley.

Eborn, J., Tummescheit, H. and K. Prölß, 2005. Air conditioning - a modelica library for dynamic simulation of AC Systems. In: Proceedings of the Fourth International Modelica Conference, March 7-8, Hamburg-Harburg, Germany, 2005, pp: 185-192.

Forster, H.K. and N. Zuber, 1955. Dynamics of vapour bubble growth and boiling heat transfer. AIChE Journal 1955;1: 531-535.

Gado, A., Hwang. Y. and R. Radermacher, 2008. Dynamic Behavior of Mobile Air-Conditioning Systems. HVAC\&R Research 14(2): 307-321.

Gnielinski, V. 1976. New equations for heat and mass transfer in turbulent pipe and channel flow. International Chemical Engineering 16: 359-368.

Gordon, J.M. and K.C. Ng, 2000. Cool Thermodynamics. International Science Publishing: Cambridge.

Grald, E.W. and J.W. MacArthur, 1992. A moving-boundary formulation for modeling time-dependent twophase flows. International Journal of Heat and Fluid Flow 13(3): 266-272.

Haberschill, P., Gay, L., Aubouin, P. and M. Lallemand, 2003. Dynamic Model of a Vapor-Compression Refrigerating Machine Using R-407C. HVAC\&R Research 9(4): 451-566.

Incropera, F.P. and D.P. DeWitt, 1996. Fundamentals of Heat and Mass Transfer, 4nt Edition, John Wiley\&Sons.

Jakobsen, A., Antonius, J. and H.J. Hagaard-Knudsen, 1999. Experimental evaluation of the use of homogeneous and slip-flow two-phase dynamic models in evaporator modeling. In: Proceedings of the 20th International Congress of Refrigeration (Sydney) 1999. Paper no. 135. 
Lemmon, E.W., Huber, M.L. and M.O. McLinden, 2007. REFPROP, NIST Standard Reference Database 23, v.8. National Institute of Standards, Gaithersburg, MD, USA.

Li, P., Qiao, H., Li ,Y., Seem J.E., Winkler J. and X. Li, 2014. Recent advances in dynamic modeling of HVAC equipment. Part 1: Equipment modelling. HVAC\&R Research 20(1): 136-149.

Limperich, D., Braun, M., Schmitz, G. and K. Prölß, 2005. System simulation of automotive refrigeration cycles. In: Proceedings of the Fourth International Modelica Conference, March 7-8, Hamburg-Harburg, Germany 2005, pp: 193-199.

MacArthur, J.W. and E.W. Grald, 1989. Unsteady compressible two-phase flow model for predicting cyclic heat pump performance and a comparison with experimental data. International Journal of Refrigeration 12(1): 2941.

Milián, V., Navarro-Esbrí, J., Ginestar, D., Molés, F. and B., Peris, 2013. Dynamic model of a shell-and-tube condenser. Analysis of the mean void fraction correlation influence on the model performance. Energy 59: 521 533.

Navarro-Esbrí, J., Ginestar, D., Belman, J.M., Milián, V. and G. Verdú, 2010. Application of a lumped model for predicting energy performance of a variable-speed vapour compression system. Applied Thermal Engineering 30(4): 286-294.

Navarro-Esbrí, J., Molés, F., Peris, B., Barragán-Cervera, A., Mendoza-Miranda, J.M., Mota-Babiloni, A. and J.M. Belman, 2014. Shell-and-tube evaporator model performance with different two-phase flow heat transfer correlations. Experimental analysis using R134a and R1234yf. Applied Thermal Engineering 62(1):80-89.

Rasmussen, B.P. 2005. Dynamic Modeling and Advanced Control of Air Conditioning and Refrigeration Systems, Dept. of Mechanical Engineering, University of Illinois.

Rasmussen, B.P. 2012. Dynamic modeling for vapor compression systems - I: Literature review. HVAC\&R Research 18(5): 934-955.

Rasmussen, B.P. and B. Shenoy, 2012. Dynamic modeling for vapor compression systems_Part II: Simulation tutorial. HVAC\&R Research 18(5): 956-973.

Rice, C.K. 1987. The effect of void fraction correlation and the heat flux assumption on refrigerant charge inventory predictions. ASHRAE Transactions 93(1) pp: 341-367.

Roetzel, W. and Y. Xuan, 1999. Dynamic Behaviour of Heat Exchangers, Vol.3. WITPress/Computational Mechanics Publications. 
Saiz Jabardo, J.M., Gonzales Mamani, W. and M.R. Ianella, 2002. Modeling and experimental evaluation of an automotive air conditioning system with a variable capacity compressor. International Journal of Refrigeration 25: $1157-1172$.

Thome, J.R. 2004. Wolverine Heat Transfer Engineering Data book III, Wolverine Tube Inc.

Wallis, G.B. 1969. One-dimensional two-phase flow. McGraw-Hill.

Wedekind, G.L., Bhatt, B.L. and B.T. Beck, 1978. A system mean void fraction model for predicting various transient phenomena associated with two phase evaporating and condensing flows. International Journal of Multiphase Flow 4: 97-114.

Willatzen, M., Pettit, N.B.O.L. and L. Ploug-Sørensen, 1998. A general dynamic simulation model for evaporators and condensers in refrigeration, Part I: moving-boundary formulation of two-phase flows with heat exchange. International Journal of Refrigeration 21: 398 - 414.

Wilson, M.J., Newell, T.A. and J.C. Chato, 1998. Experimental Investigation of Void Fraction during Horizontal Flow in Larger Diameter Refrigeration Applications, Air Conditioning and Refrigeration Center, University of Illinois at Urbana Champaign. ACRC TR-140.

Woldesemayat, M.A. and A.J. Ghajar, 2007. Comparison of void fraction correlations for different flow patterns in horizontal and upward inclined pipes. International Journal of Multiphase Flow 33: 347-370.

Zhang, W.J. and C.L. Zhang, 2006. A generalized moving boundary model for transient simulation of dryexpansion evaporators under larger disturbances. International Journal of Refrigeration 29: 1119-1127.

Zukauskas, A.A. 1987. Convective heat transfer in cross flow. In Handbook of Single-Phase Convective Heat Transfer, Kakac S, Shah RK, Aung W (eds). Wiley: New York, 6.1-6.45. 


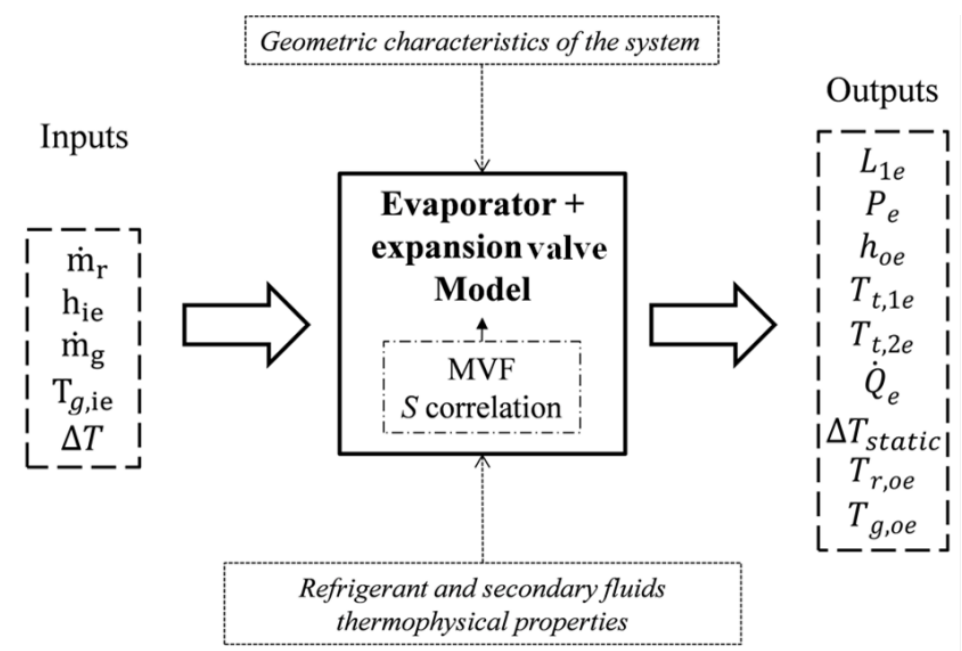

Fig. 1. Model scheme. 




(a)



(b)

Fig. 2. Shell-and-tube evaporator inner structure and fluid path (a) and equivalent axial tube with two evaporator zones (b). 


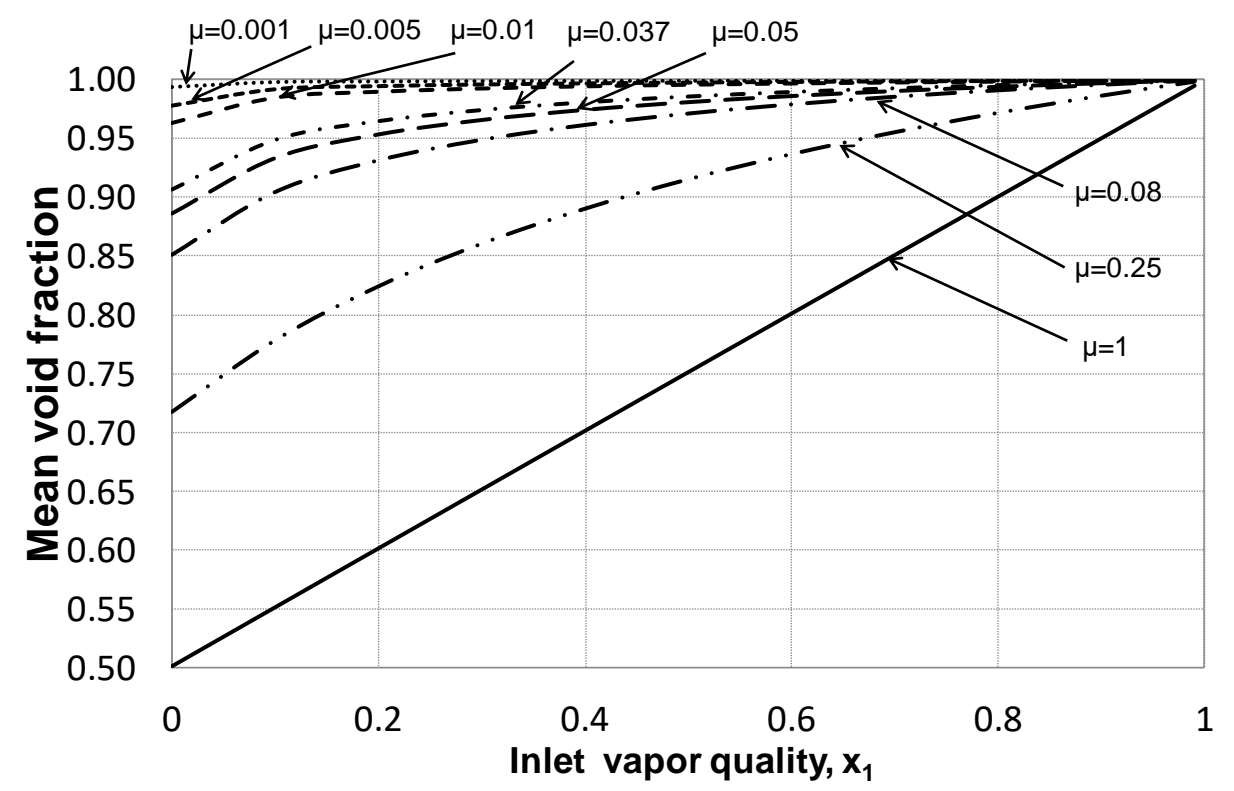

Fig. 3. Quality based mean void fraction, $S=S_{Z}$. 


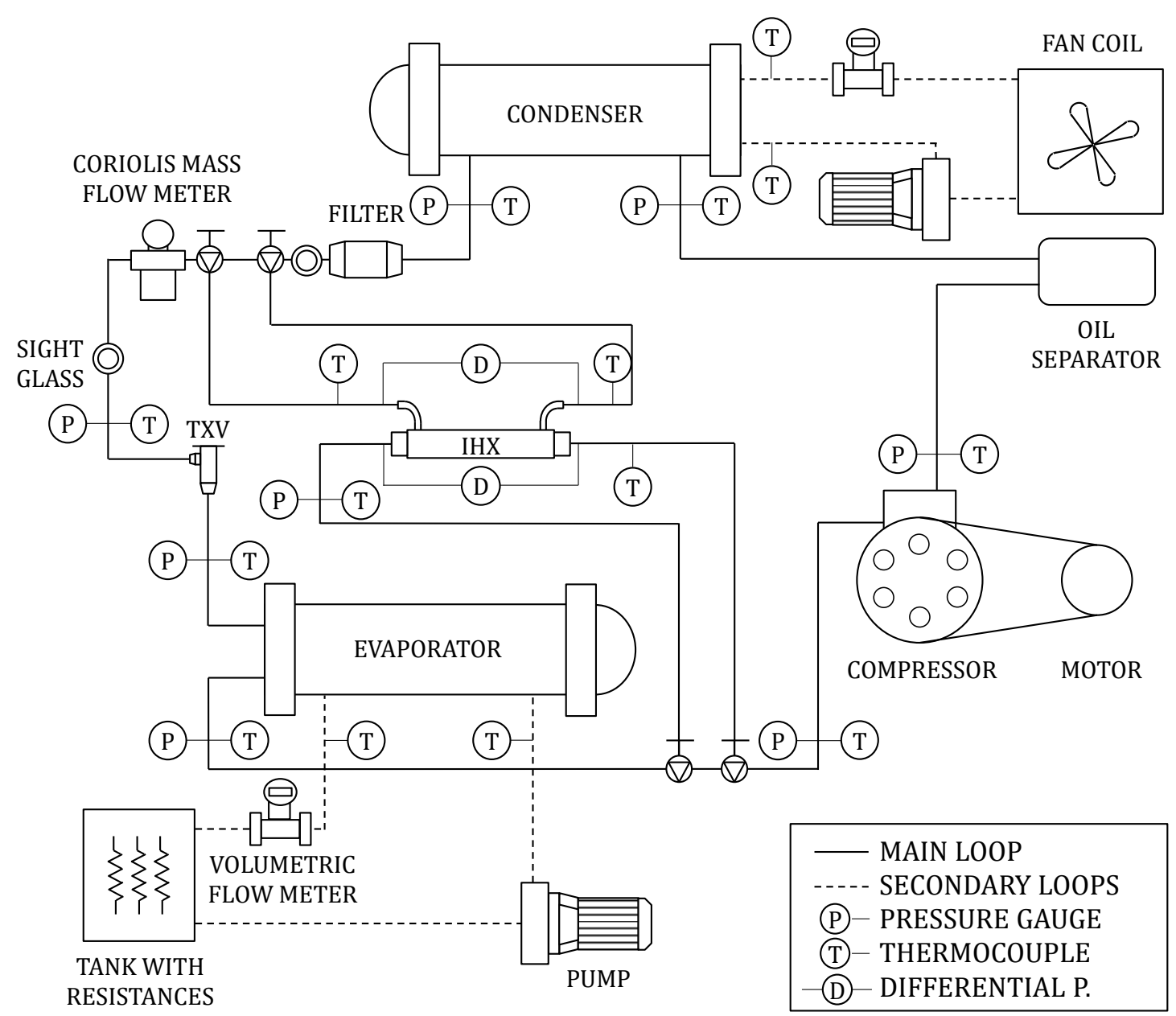

Fig. 4. Simplified vapour compression plant scheme. 


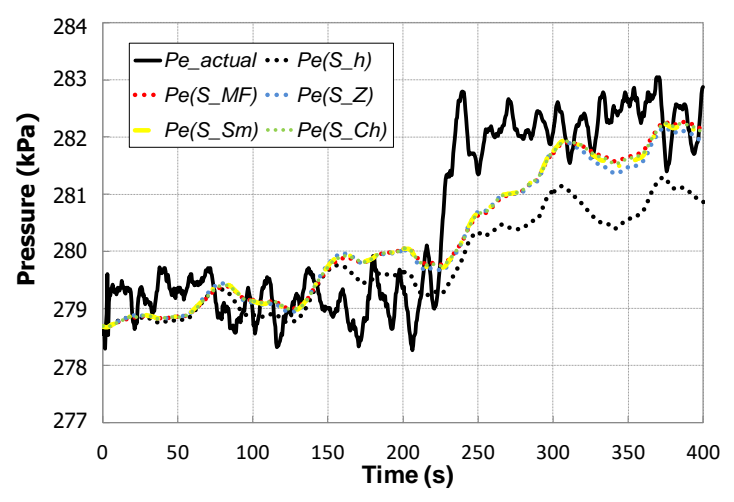

(a)

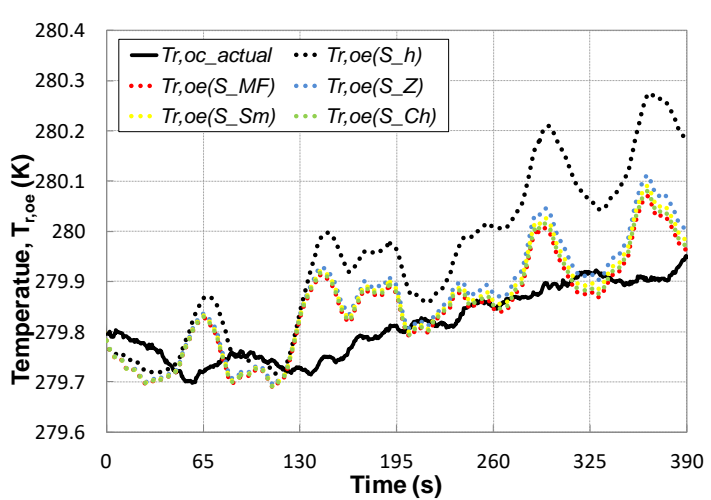

(b)

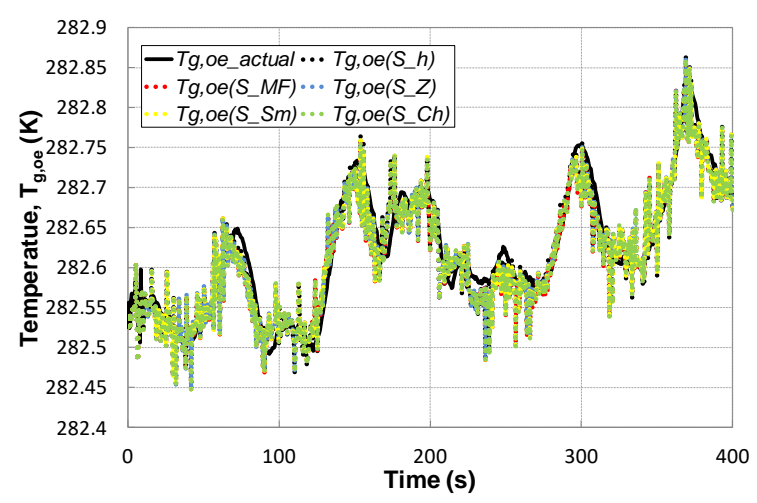

(c)

Fig. 5. Test 1. Model outputs due to small decrease in refrigerant mass flux, (a) evaporating pressure, (b) refrigerant outlet temperature, (c) secondary fluid outlet temperature. 


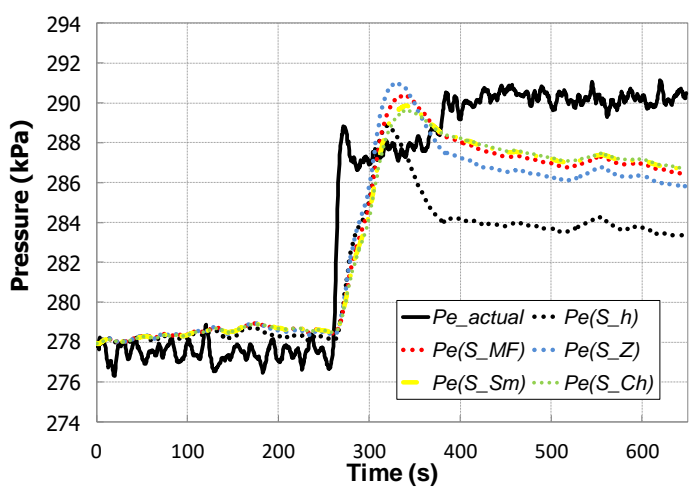

(a)

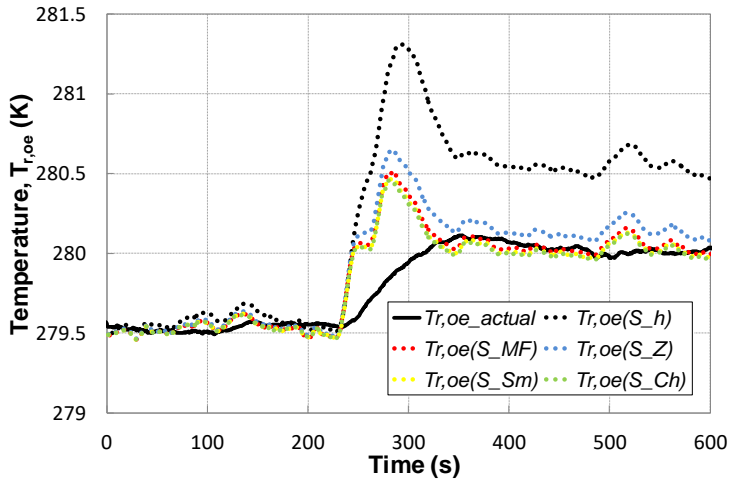

(b)



(c)

Fig. 6. Test 3. Model outputs due to medium decrease refrigerant in mass flux, (a) evaporating pressure, (b) refrigerant outlet temperature, (c) secondary fluid outlet temperature. 


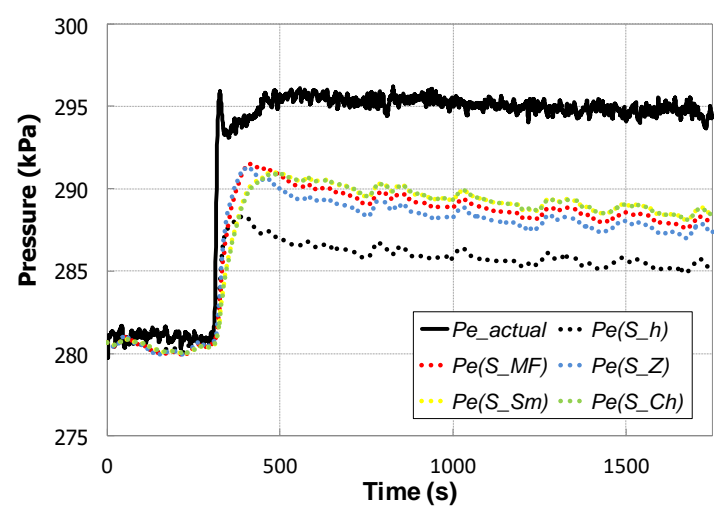

(a)

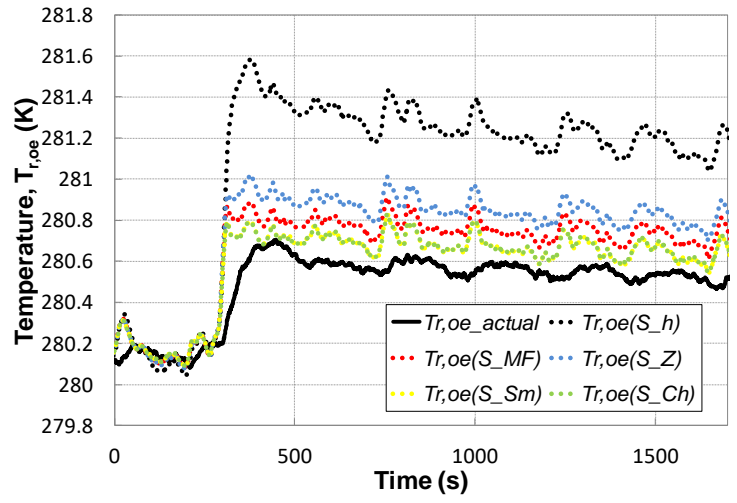

(b)



(c)

Fig. 7. Test 5. Model outputs due to high decrease in refrigerant mass flux, (a) evaporating pressure, (b) refrigerant outlet temperature, (c) secondary fluid outlet temperature. 


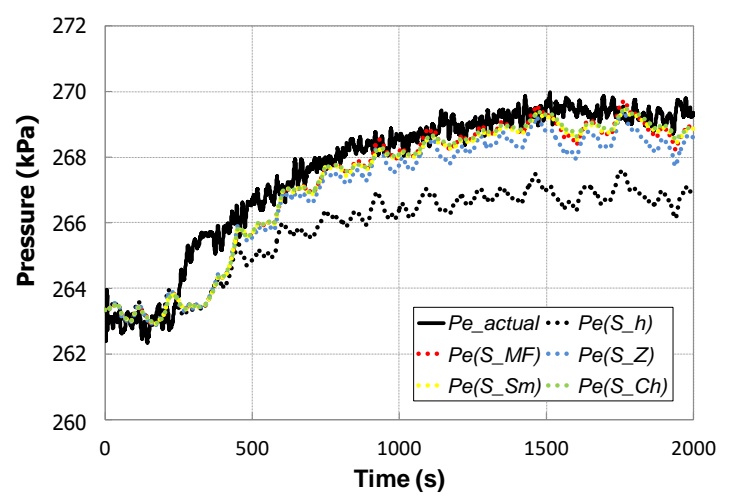

(a)



(b)

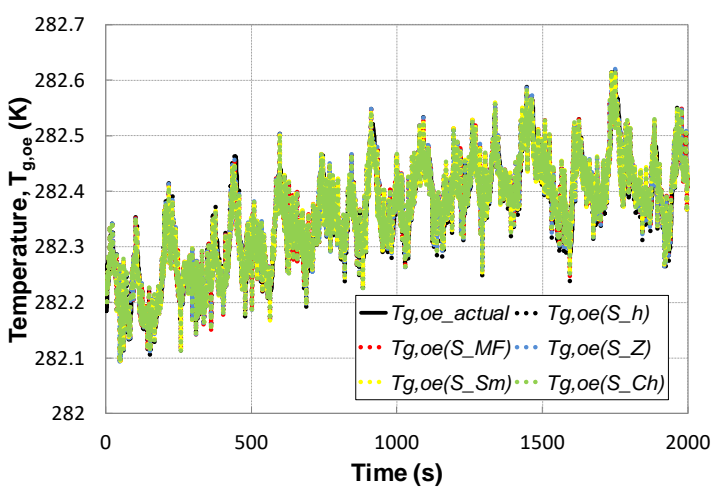

(c)

Fig. 8. Test 7. Model outputs due to small decrease in evaporator inlet enthalpy, (a) evaporating pressure, (b) refrigerant outlet temperature, (c) secondary fluid outlet temperature. 


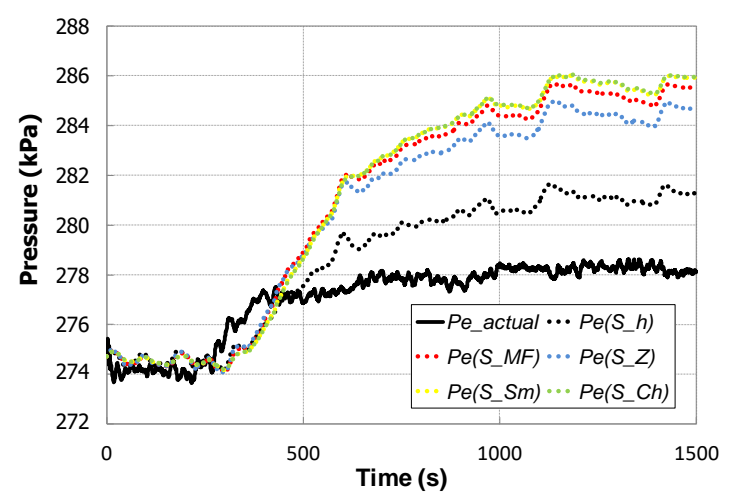

(a)



(b)

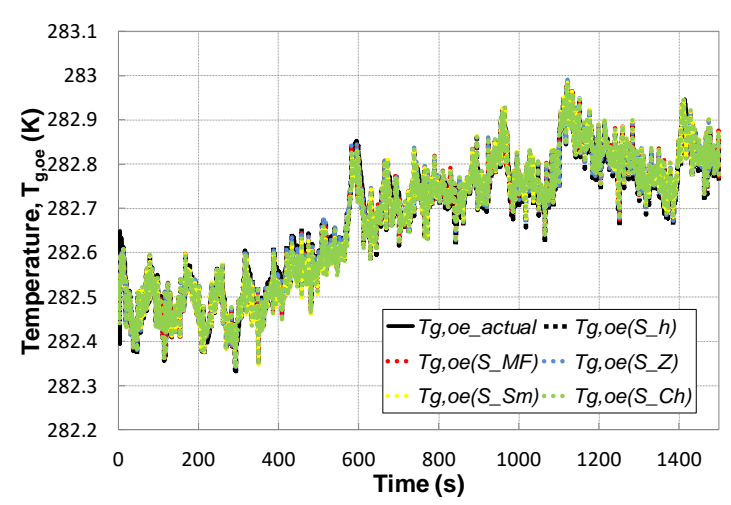

(c)

Fig. 9. Test 9. Model outputs due to high decrease in evaporator inlet enthalpy, (a) evaporating pressure, (b) refrigerant outlet temperature, (c) secondary fluid outlet temperature. 


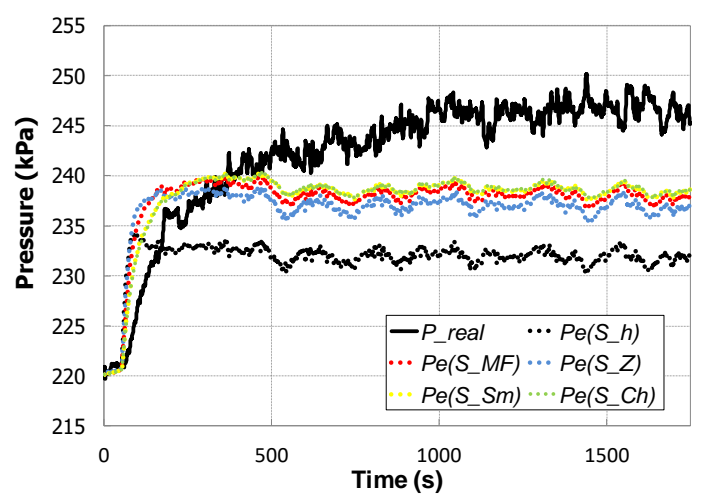

(a)

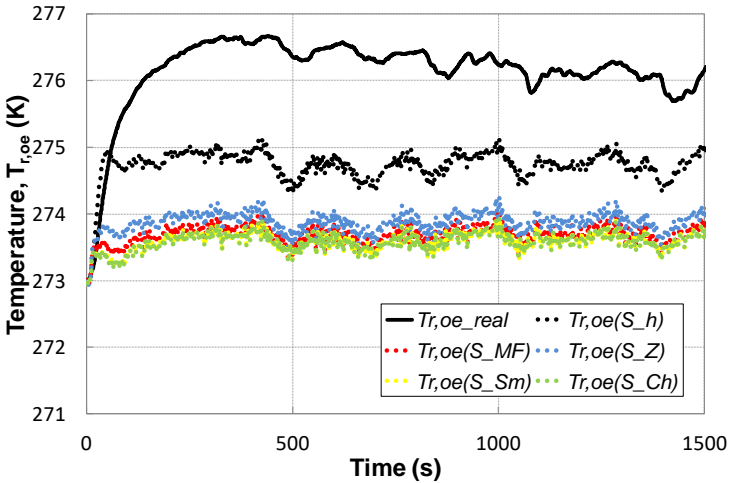

(b)



(c)

Fig. 10. Test 11. Model outputs due to sharp decrease in evaporator secondary fluid flow, (a) evaporating pressure, (b) refrigerant outlet temperature, (c) secondary fluid outlet temperature. 


\section{FIGURE CAPTIONS}

Fig. 1. Model scheme.

Fig. 2. Shell-and-tube evaporator inner structure and fluid path (a), and equivalent axial tube with two evaporator zones (b).

Fig. 3. Quality based mean void fraction, $S=S_{Z}$.

Fig. 4. Simplified vapour compression plant scheme.

Fig. 5. Test 1. Model outputs due to small decrease in refrigerant mass flux, (a) evaporating pressure, (b) refrigerant outlet temperature, (c) secondary fluid outlet temperature.

Fig. 6. Test 3. Model outputs due to medium decrease in refrigerant mass flux decrease, (a) evaporating pressure, (b) refrigerant outlet temperature, (c) secondary fluid outlet temperature.

Fig. 7. Test 5. Model outputs due to high decrease in refrigerant mass flux decrease, (a) evaporating pressure, (b) refrigerant outlet temperature, (c) secondary fluid outlet temperature.

Fig. 8. Test 7. Model outputs due to small decrease in evaporator inlet enthalpy, (a) evaporating pressure, (b) refrigerant outlet temperature, (c) secondary fluid outlet temperature. 
Fig. 9. Test 9. Model outputs due to high decrease in evaporator inlet enthalpy, (a) evaporating pressure, (b) refrigerant outlet temperature, (c) secondary fluid outlet temperature.

Fig. 10. Test 11. Model outputs due to sharp decrease in evaporator secondary fluid flow, (a) evaporating pressure, (b) refrigerant outlet temperature, (c) secondary fluid outlet temperature. 
Table 1 . Terms $z_{i j}$ in system model.

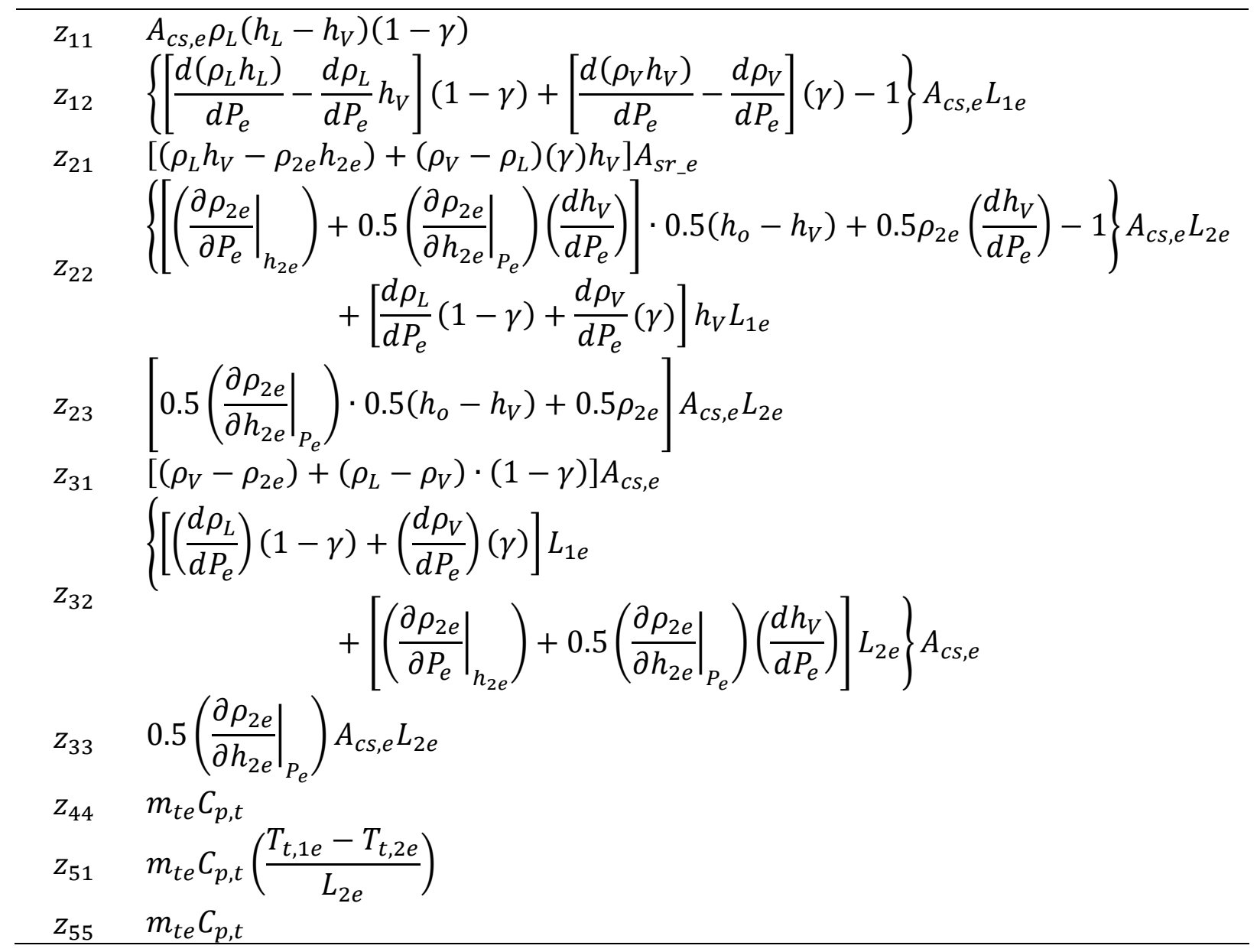


Table 2. Terms $y_{i j}$ in system model.

$$
\begin{array}{ll}
y_{11} & \dot{m}_{r}\left(h_{i}-h_{V}\right)+\alpha_{i n, 1 e} \cdot A_{i n, e}\left(\frac{L_{1 e}}{L_{t e}}\right)\left(T_{t, 1 e}-T_{r, 1 e}\right) \\
y_{21} & \dot{m}_{r} h_{V}-\dot{m}_{r} h_{o}+\alpha_{i n, 2 e} \cdot A_{i n, e} \frac{L_{2 e}}{L_{t e}}\left(T_{t, 2 e}-T_{r, 2 e}\right) \\
y_{31} & 0 \\
y_{41} & \alpha_{e x, 1 e} A_{e x, e} \frac{L_{1 e}}{L_{t e}}\left(T_{g, 1 e}-T_{t, 1 e}\right)-\alpha_{i n, 1 e} A_{i n, e}\left(\frac{L_{1 e}}{L_{t e}}\right)\left(T_{t, 1 e}-T_{r, 1 e}\right) \\
y_{51} & \alpha_{e x, 2 e} A_{e x, e} \frac{L_{2 e}}{L_{t e}}\left(T_{g, 2 e}-T_{t, 2 e}\right)-\alpha_{i n, 2 e} A_{i n, e} \frac{L_{2 e}}{L_{t e}}\left(T_{t, 2 e}-T_{r, 2 e}\right)
\end{array}
$$


Table 3. Equations used to obtain the heat transfer coefficients.

\begin{tabular}{|c|c|c|}
\hline $\begin{array}{l}\text { Heat transfer } \\
\text { coefficient }\end{array}$ & Correlation & Equations \\
\hline \multirow{2}{*}{$\begin{array}{l}\text { Shell side: } \\
\text { both regions }\end{array}$} & \multirow{2}{*}{$\begin{array}{l}\text { Zukauskas } \\
\text { (1987) }\end{array}$} & $\alpha_{e x,(1 e, 2 e)}=\frac{k_{g}}{D_{e x}} \cdot C \cdot R e_{\text {max }}^{m} \cdot P r_{g}^{0.36} \cdot\left(\frac{P r_{g}}{P r_{g, t}}\right)^{0.25}$ \\
\hline & & $\begin{array}{c}C \text { and } m \text { depends on the configuration and } R e_{D} \\
\text { (Incropera and DeWitt, 1996) }\end{array}$ \\
\hline \multirow{8}{*}{$\begin{array}{l}\text { Tube side: } \\
\text { evaporating } \\
\text { region }\end{array}$} & \multirow{8}{*}{ Chen (1966) } & $\alpha_{i n, 1 e}=s f \alpha_{n b}+F \alpha_{c o n v}$ \\
\hline & & 1 \\
\hline & & $s f=\overline{1+0.00000253 \operatorname{Re}_{b f}^{1.17}}$ \\
\hline & & where $R e_{b f}=R e_{L} \cdot F^{1.25}$ \\
\hline & & $\int \quad 1$, if $\frac{1}{X_{t t}} \leq 0.1$ \\
\hline & & $F=\left\{\begin{array}{l}2.35\left(\frac{1}{X_{t t}}+0.213\right)^{0.736} \text {, if } \frac{1}{X_{t t}}>0.1\end{array}\right.$ \\
\hline & & $\begin{array}{c}\alpha_{n b}=0.00122\left[\frac{k_{L}^{0.79} c_{p L}^{0.45} \rho_{L}^{0.49}}{\sigma^{0.5} u_{L}^{0.29} h_{L V}^{0.24} \rho_{V}^{0.24}}\right] \Delta T_{s a t}^{0.24} \Delta P_{s a t}^{0.75} \text { (Forster } \\
\text { and Zuber, 1955) }\end{array}$ \\
\hline & & $\alpha_{\text {conv }}=0.023 R e_{L}^{0.8} \operatorname{Pr}_{L}^{0.4}\left(\frac{k_{L}}{D_{\text {in }}}\right)$ (Dittus and Boelter, \\
\hline \multirow{2}{*}{$\begin{array}{l}\text { Tube side: } \\
\text { superheating } \\
\text { region }\end{array}$} & \multirow{2}{*}{$\begin{array}{l}\text { Gnielinski } \\
\text { (1976) }\end{array}$} & $=\frac{k_{r, 2 e}}{\left(\frac{f_{2 e}}{8}\right)\left(R e_{2 e}-1000\right) P r_{2 e}}$ \\
\hline & & $\alpha_{i n, 2 e}=\overline{D_{i n}} \overline{1+12.7\left(\frac{f_{2 e}}{8}\right)^{1 / 2}\left(\operatorname{Pr}_{2 e}^{2 / 3}-1\right)}$ \\
\hline
\end{tabular}


Table 4. Tube characteristics.

Number

$D_{\text {in }} / D_{\text {ex }}$

Thickness of inner microfins

Total length

External exchange surface

Tube-side volume

Shell-side volume
76

$8.22 \times 10^{-3} / 9.52 \times 10^{-3} \mathrm{~m}$

$0.2 \times 10^{-3} \mathrm{~m}$

$0.92 \mathrm{~m}$

$1.81 \mathrm{~m}^{2}$

$3.3 \times 10^{-3} \mathrm{~m}^{3}$

$8 \times 10^{-3} \mathrm{~m}^{3}$ 
Table 5. Measured parameters and equipment uncertainty.

\begin{tabular}{llc}
\hline Measured parameters & Sensor & Uncertainty \\
\hline Temperatures & K-type thermocouples & $\pm 0.3 \mathrm{~K}$ \\
Pressures & Piezoelectric pressure transducers & $\pm 0.1 \%$ \\
Mass flow rate & Coriolis mass flow meter & $\pm 0.22 \%$ \\
Compressor power consumption & Digital wattmeter & $\pm 0.5 \%$ \\
Rotation speed & Capacitive sensor & $\pm 1 \%$ \\
Volumetric flow rate & Electromagnetic flow meters & $\pm 0.33 \%$ \\
\hline
\end{tabular}


Table 6. Changes of parameters that originate the transients.

\begin{tabular}{ccccc}
\hline Test & $\dot{m}_{r}\left(\mathrm{~kg} \mathrm{~s}^{-1}\right)$ & $h_{i, a v g}\left(\mathrm{~kJ} \mathrm{~kg}^{-1}\right)$ & $\dot{V}_{g}\left(\mathrm{~m}^{3} \mathrm{~s}^{-1}\right)$ & $T_{g, i}(\mathrm{~K})$ \\
\hline Test 1 & $0.0563 \rightarrow 0.0548$ & $256.6( \pm 0.1)$ & $8.210^{-4}\left( \pm 1.410^{-6}\right)$ & $285.16( \pm 0.08)$ \\
Test 2 & $0.0555 \rightarrow 0.0569$ & $259.7( \pm 0.2)$ & $8.210^{-4}\left( \pm 2.810^{-6}\right)$ & $285.09( \pm 0.06)$ \\
Test 3 & $0.0556 \rightarrow 0.0503$ & $248.7( \pm 0.9)$ & $8.210^{-4}\left( \pm 2.810^{-6}\right)$ & $285.11( \pm 0.07$ \\
Test 4 & $0.0483 \rightarrow 0.0543$ & $266.8( \pm 1.5)$ & $8.210^{-4}\left( \pm 1.410^{-6}\right)$ & $285.19( \pm 0.05)$ \\
Test 5 & $0.0569 \rightarrow 0.0497$ & $257.4( \pm 2.1)$ & $8.210^{-4}\left( \pm 2.810^{-6}\right)$ & $285.11( \pm 0.06)$ \\
Test 6 & $0.0497 \rightarrow 0.0603$ & $261.0( \pm 3.0)$ & $8.210^{-4}\left( \pm 2.810^{-6}\right)$ & $285.08( \pm 0.07)$ \\
Test 7 & $0.0599( \pm 0.0003)$ & $251 \rightarrow 258$ & $8.210^{-4}\left( \pm 1.710^{-6}\right)$ & $285.10( \pm 0.07)$ \\
Test 8 & $0.0553( \pm 0.0003)$ & $256 \rightarrow 251$ & $8.210^{-4}\left( \pm 2.210^{-6}\right)$ & $285.14( \pm 0.07)$ \\
Test 9 & $0.0591( \pm 0.0007)$ & $264 \rightarrow 273$ & $8.210^{-4}\left( \pm 2.810^{-6}\right)$ & $285.15( \pm 0.07)$ \\
Test 10 & $0.0552( \pm 0.0005)$ & $270 \rightarrow 260$ & $8.210^{-4}\left( \pm 2.510^{-6}\right)$ & $285.14( \pm 0.07)$ \\
Test 11 & $0.0546( \pm 0.0031)$ & $242.0( \pm 1.1)$ & $3.310^{-4} \rightarrow 5.610^{-4}$ & $284.76( \pm 0.16)$ \\
Test 12 & $0.0489( \pm 0.0030)$ & $234.1( \pm 1.1)$ & $5.610^{-4} \rightarrow 3.310^{-4}$ & $284.56( \pm 0.21)$ \\
\hline
\end{tabular}


Table 7. RMS values.

\begin{tabular}{|c|c|c|c|c|c|c|}
\hline & & $S_{h}$ & $S_{M F}$ & $S_{Z}$ & $S_{S m}$ & $S_{C h}$ \\
\hline \multirow[t]{3}{*}{ Test 1} & $P_{e}$ & 1273 & 943 & 967 & 951 & 945 \\
\hline & $T_{r, o e}$ & 0.1734 & 0.0772 & 0.0908 & 0.0835 & 0.0799 \\
\hline & $T_{g, o e}$ & 0.0465 & 0.0470 & 0.0467 & 0.0468 & 0.0469 \\
\hline \multirow[t]{3}{*}{ Test 2} & $P_{e}$ & 918 & 737 & 765 & 759 & 764 \\
\hline & $T_{r, o e}$ & 0.0917 & 0.0734 & 0.0715 & 0.0757 & 0.0758 \\
\hline & $T_{g, o e}$ & 0.0502 & 0.0497 & 0.0501 & 0.0483 & 0.0482 \\
\hline \multirow[t]{3}{*}{ Test 3} & $P_{e}$ & 4527 & 2731 & 3047 & 2643 & 2622 \\
\hline & $T_{r, o e}$ & 0.5365 & 0.1654 & 0.2153 & 0.1553 & 0.1522 \\
\hline & $T_{g, o e}$ & 0.0610 & 0.0724 & 0.0678 & 0.0745 & 0.0754 \\
\hline \multirow[t]{3}{*}{ Test 4} & $P_{e}$ & 4339 & 2273 & 2657 & 2121 & 2124 \\
\hline & $T_{r, o e}$ & 0.957 & 0.097 & 0.158 & 0.071 & 0.070 \\
\hline & $T_{g, o e}$ & 0.048 & 0.051 & 0.049 & 0.055 & 0.056 \\
\hline \multirow[t]{3}{*}{ Test 5} & $P_{e}$ & 8236 & 5598 & 6085 & 5338 & 5356 \\
\hline & $T_{r, o e}$ & 0.6396 & 0.1958 & 0.2783 & 0.1305 & 0.1310 \\
\hline & $T_{g, o e}$ & 0.0478 & 0.0509 & 0.0492 & 0.0551 & 0.0554 \\
\hline \multirow[t]{3}{*}{ Test 6} & $P_{e}$ & 8106 & 4114 & 4818 & 4065 & 3939 \\
\hline & $T_{r, o e}$ & 0.7656 & 0.1921 & 0.2219 & 0.1942 & 0.1940 \\
\hline & $T_{g, o e}$ & 0.073 & 0.093 & 0.083 & 0.091 & 0.094 \\
\hline \multirow[t]{3}{*}{ Test 7} & $P_{e}$ & 2105 & 857 & 1021 & 860 & 838 \\
\hline & $T_{r, o e}$ & 0.183 & 0.119 & 0.087 & 0.115 & 0.123 \\
\hline & $T_{g, o e}$ & 0.0446 & 0.0448 & 0.0447 & 0.0448 & 0.0448 \\
\hline \multirow[t]{3}{*}{ Test 8} & $P_{e}$ & 768 & 1356 & 1209 & 1325 & 1349 \\
\hline & $T_{r, o e}$ & 0.2722 & 0.1112 & 0.1351 & 0.1156 & 0.1115 \\
\hline & $T_{g, o e}$ & 0.6879 & 0.5179 & 0.5499 & 0.5238 & 0.5177 \\
\hline \multirow[t]{3}{*}{ Test 9} & $P_{e}$ & 2146 & 5011 & 4453 & 5254 & 5272 \\
\hline & $T_{r, o e}$ & 0.7908 & 0.3276 & 0.4192 & 0.2821 & 0.2771 \\
\hline & $T_{g, o e}$ & 0.0450 & 0.0447 & 0.0444 & 0.0455 & 0.0457 \\
\hline \multirow[t]{3}{*}{ Test 10} & $P_{e}$ & 1299 & 3813 & 3297 & 4069 & 4082 \\
\hline & $T_{r, o e}$ & 0.2985 & 0.1553 & 0.0858 & 0.2051 & 0.2085 \\
\hline & $T_{g, o e}$ & 0.0432 & 0.0429 & 0.0428 & 0.0431 & 0.0432 \\
\hline \multirow[t]{3}{*}{ Test 11} & $P_{e}$ & 11950 & 6713 & 7671 & 6147 & 6089 \\
\hline & $T_{r, o e}$ & 1.3698 & 2.4007 & 2.2173 & 2.4897 & 2.4976 \\
\hline & $T_{g, o e}$ & 0.2384 & 0.2478 & 0.2451 & 0.2504 & 0.2507 \\
\hline \multirow[t]{3}{*}{ Test 12} & $P_{e}$ & 15201 & 13208 & 13258 & 13539 & 13631 \\
\hline & $T_{r, o e}$ & 2.711 & 3.381 & 3.248 & 3.442 & 3.451 \\
\hline & $T_{g, o e}$ & 0.5768 & 0.5690 & 0.5725 & 0.5669 & 0.5666 \\
\hline
\end{tabular}

